Article

\title{
Fabrication of a Multi-Walled Nanotube (MWNT) Ionic Liquid Electrode and Its Application for Sensing Phenolics in Red Wines
}

\author{
Kyo-Il Kim, Hee-Young Kang, Jae-Chan Lee and Seong-Ho Choi * \\ Department of Chemistry, Hannam University, Daejeon 305-811, Republic of Korea; \\ E-Mails: sinjangkki@naver.com (K.K); dartpip@nate.com (H.K); jclee@hnu.kr (J.L) \\ * Author to whom correspondence should be addressed; E-Mail: shchoi@hnu.kr; \\ Tel.: +82-42-629-8824; Fax: +82-42-629-8811.
}

Received: 16 June 2009; in revised form: 14 July 2009 / Accepted: 18 August 2009 /

Published: 26 August 2009

\begin{abstract}
A multi-walled nanotube (MWNT) ionic liquid was prepared by the immobilization of 1-butylimidazole bromide onto an epoxy group on a poly(glycidyl methacrylate)-grafted MWNT, which was synthesized by radiation-induced graft polymerization of glycidyl methacrylate onto MWNT in an aqueous solution. Subsequently, a MWNT ionic liquid electrode was fabricated by hand-casting MWNT ionic liquid, tyrosinase, and chitosan solution as a binder on indium tin oxide (ITO) glass. The sensing ranges of the MWNT ionic liquid electrode with immobilized tyrosinase was in the range of $0.01-0.08 \mathrm{mM}$ in a phosphate buffer solution. The optimal conditions such as $\mathrm{pH}$, temperature, and effects of different phenolic compounds were determined. The total phenolic compounds of three commercial red wines were also determined on the tyrosinase-immobilized biosensor.
\end{abstract}

Keywords: MWNT ion liquid electrode; glycidyl methacrylate; radiation-induced graft polymerization; phenolics; red wines

\section{Introduction}

A great number of papers on carbon nanotube (CNT)-based sensors have been published over the past several years mainly because CNTs have the following advantages for electrochemical sensor applications: (1) small size with a large surface area, (2) high sensitivity, (3) fast response time, (4) 
enhanced electron transfer and (5) easy protein immobilization on CNT-modified electrodes, coupled with the fact that several methods have been developed [1-3].

In recent years, direct electrochemistry of biologically important enzymes has been studied with ionic liquids in both theoretical and practical applications because ionic liquids, with their high polarity, non-coordination power, high selectivity, fast rates and great enzyme stability are considered to be suitable media for supporting biocatalytic processes [4-5]. Enzymes are usually active and protein refolding is improved in ionic liquids [6]. Zeng and coworkers fabricated a modified GC electrode by entrapping glucose oxidase (GOx) in a nano $\mathrm{Au}$ particle-ionic liquid- $N, N$ dimethylformamide composite film [7]. Sun et al. published information about the fabrication of a modified imidazolium-based carbon ionic liquid electrode by entrapping $\mathrm{Hb}$ into a sodium alginate hydrogel film [8]. Safavi et al. constructed a modified pyridium-based carbon ionic liquid electrode using octylpyridinium chloride ([OCPy] $[\mathrm{Cl}]$ ) to immobilize direct hemoglobin $(\mathrm{Hb})$ on carbon ionic liquid electrode (CILE) [9]. However, to the best of our knowledge, no papers on CNT ion liquid electrodes for the immobilization of enzymes have been published until now, because it is very difficult for the ion liquids to be introduced onto a CNT surface.

Radiation-induced graft polymerization (RIGP) is a useful method for the introduction of functional groups into different polymer matrixes using specially selected monomers. There have been several reports on radiation-induced graft polymerization of polar monomers onto polymer substrates with hydrophobic properties to obtain hydrophilic properties for versatile applications [10-11]. The RIGP method can be easily modified for the surface of MWNTs. In a previous paper [13], a MWNT was modified by various vinyl monomers in an aqueous solution at room temperature by RIGP. In particular, the epoxy groups of poly(glycidyl methacrylate) [poly(GMA)] are changeable to alcohols [14], amines [15], phosphonic acids [16], sulfonic acids [17], etc [18]. However, little has been reported about the introduction of ionic liquids onto an epoxy group of a grafted poly(GMA).

Wines, particularly red wines, contain numerous biologically active compounds, the most important of which are polyphenols. The nutritional importance of polyphenols is attributed to their antioxidant properties and there has been increasing interest in the flavonoids and related phenolics which are naturally found in red wines [19]. Red wines have been reported to be preventive of many ailments and may possibly play a role in reducing thrombotic and anthrogenic processes. In addition, polyphenols also contribute substantially to the quality of wines, affecting their color, flavor, stability and aging behavior [20]. However, little has been reported regarding the easy determination of the total amount of phenolics in red wine by an electrochemical method.

In this study, we synthesized poly(GMA)-grafted MWNT by RIGP of GMA in an aqueous solution in order to immobilize imidazole bromide as an ionic liquid. A MWNT ionic liquid electrode was prepared on an ITO electrode by hand casting of MWNT ionic liquid, tyrosinase, and chitosan as a binder. The sensing efficiency of the prepared MWNT ionic liquid electrode for phenol was evaluated in a phosphate buffer solution. The optimal conditions such as $\mathrm{pH}$, temperature, and effects of different phenolic compounds were evaluated. Total phenolic compounds for three commercial red wines were also analysed using the prepared enzyme electrode. 


\section{Experimental}

\subsection{Reagents}

Tyrosinase from mushrooms (EC 1.14.18.1), phenol, $p$-cresol, catechol, glycidyl methacrylate and chitosan were purchased from the Sigma-Aldrich Korea Ltd. Company. An ITO electrode as a working electrode (working area $0.7 \times 1.1 \mathrm{~cm}^{2}, 10 \Omega$ resistance) was purchased from Dasom RMS Co. (Korea). MWNT (CM-95) was supplied by Hanwha Nanotech Co., Ltd (Korea). Three Korean red wines (Chateau Mani-dry, Chateau Mani-sweet, and Chateau Mani-nouveau) were used in order to provide phenolics. Solutions for the experiments were prepared with water purified in a Milli-Q puls water

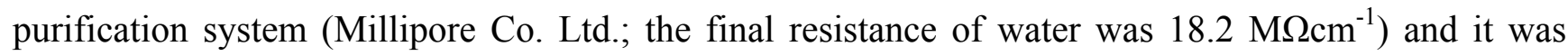
degassed prior to each measurement.

\subsection{Synthesis of the poly(GMA)-g-MWNT}

The MWNT's were purified in order to remove the catalyst and non-crystallized carbon impurities. Briefly, MWNT's were treated with a $3: 1$ (vol-\%) mixture of $\mathrm{H}_{2} \mathrm{SO}_{4} / \mathrm{HNO}_{3}$ and in the process MWNT's were cut into shorter segments. The purified and cut MWNT were used as the supporting materials for grafting with GMA. The MWNT (2.0 g) and GMA (2.0 g) were mixed in an aqueous solution $(20 \mathrm{~mL})$. Nitrogen gas was bubbled through the solution for 30 minutes to remove oxygen gas, and the solution was irradiated by $\gamma$-rays from $\mathrm{Co}-60$ source under atmospheric pressure and ambient temperature. A total irradiation dose of $30 \mathrm{kGy}$ (a dose rate $=1.0 \times 10^{4} \mathrm{~Gy} / \mathrm{h}$ ) was used. The poly(GMA)-g-MWNT's were dried in a vacuum oven at $50{ }^{\circ} \mathrm{C}$ for $8 \mathrm{hrs}$.

\subsection{Fabrication of the Enzyme Electrode Based on Carboxylic Acid-modified MWNT}

Scheme 1 shows the fabrication procedure of the MWNT ionic liquid electrode based on an epoxy group-modified MWNT prepared by $\gamma$-irradiation. A mixed solution containing chitosan (3.2 $\mathrm{mg})$ as a binder, tyrosinase $(25,000$ units, $0.3 \mathrm{mg})$, and MWNT ionic liquid (3.2 $\mathrm{mg})$ as the supporter was prepared in an acetic acid solution $(0.01 \mathrm{~mL})$, and then the mixed solution $(9.0 \mu \mathrm{L})$ was coated on the surface of a pre-cleaned ITO electrode by the hand-cast method. The prepared MWNT ion liquid electrode was kept at $4{ }^{\circ} \mathrm{C}$ until used.

\subsection{Instrumentation}

Cyclic voltammetric (CV) experiments were performed with a Potentiostat/Gavanostat model 283 (Ametek PAR, U.S.A.) or CV-50w voltametry (Bioanalytical Systems, Inc. U.S.A.). All experiments were carried out with a conventional three-electrode system. The working electrode was an ITO electrode coated with the poly(GMA)-g-MWNT, counter electrode was the platinum wire, and reference electrode was an $\mathrm{Ag} / \mathrm{AgCl}$ (sat'd $\mathrm{KCl}$ ). The surface morphology of the samples was determined using scanning electron microscopy (SEM, S-3000N, Hitachi Science System Ltd., Japan), 
and by HR-TEM (JEOL, JEM-2010, USA). The thermal gravimetric analysis (TGA) was conducted on a Scinco TGA S-1000 (Seoul, Korea) under $\mathrm{N}_{2}$ flow from $25{ }^{\circ} \mathrm{C}$ to $700{ }^{\circ} \mathrm{C}$ at a heating rate of $20{ }^{\circ} \mathrm{C} / \mathrm{min}$.

Scheme 1. Synthesis procedure of the MWNT ionic liquid for biosensors.

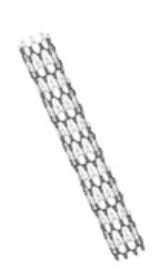

MWNT

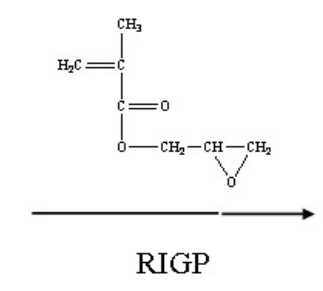

RIGP

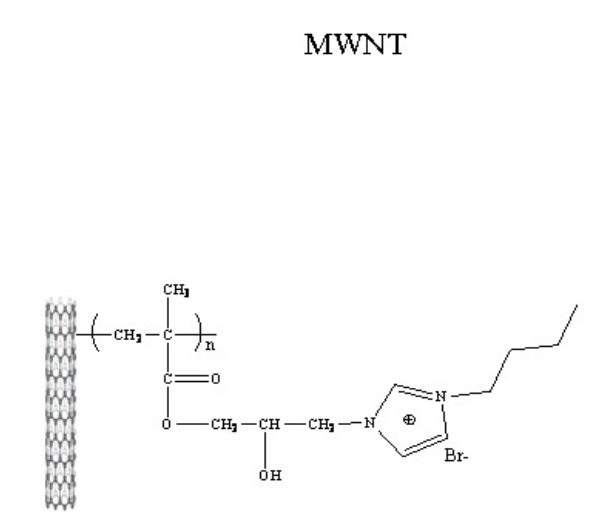

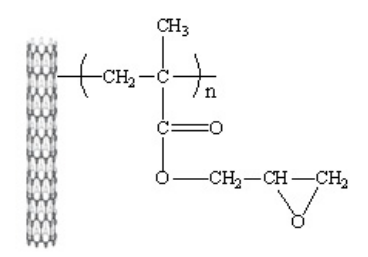

Poly(GMA)-g-MWNT
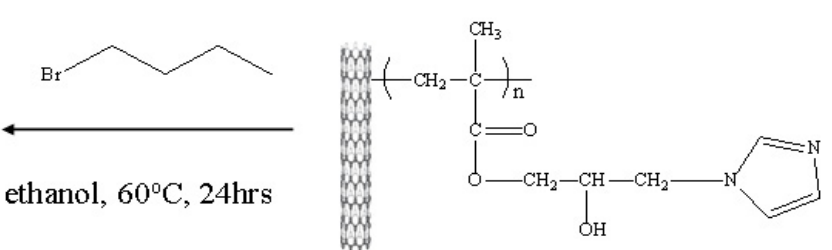

\section{Results and Discussion}

\subsection{Fabrication and Characterization of MWNT Ion Liquid Electrode}

The functionalization of MWNT is one of the most active fields in nanotubes research and it is an effective tool to broaden the electrochemical application spectrum of MWNT. We functionalized MWNT from a GMA monomer in the presence of MWNT by RIGP in an aqueous solution, in order to introduce the epoxy group. The epoxy group can be converted into an ionic liquid group such as imidazolium cations for the purpose of enhancing electron transfer. In our previous paper, the morphology, physical and chemical properties for the vinyl polymer-grafted MWNT obtained by RIGP were evaluated [13]. In order to enhance electron transfer, we introduced butyl imidazolium bromide according to Scheme 1 and carried out the corresponding elemental analysis (Table 1).

Table 1. Elemental analysis of the prepared MWNT ion liquids.

\begin{tabular}{lllll}
\hline Sample & N (\%) & $\mathbf{C ~ ( \% )}$ & H (\%) & O (\%) \\
\hline Pure MWNT & & 76.14 & 0.74 & 11.81 \\
poly(GMA)-g-MWNT & & 66.78 & 4.80 & 22.25 \\
MWNT ionic liquid & 5.35 & 55.79 & 4.63 & 18.58 \\
\hline
\end{tabular}


The content of imidazolium ion was estimated to be approximately $11.0 \%$ by the elemental analysis. This indicates that butyl imidazolium cations were successfully introduced onto an epoxy group of poly(GMA)-g-MWNT.

Figure 1. TEM images of pure MWNT (a), poly(GMA)-g-MWNT (b) and MWNT ionic liquid (c).

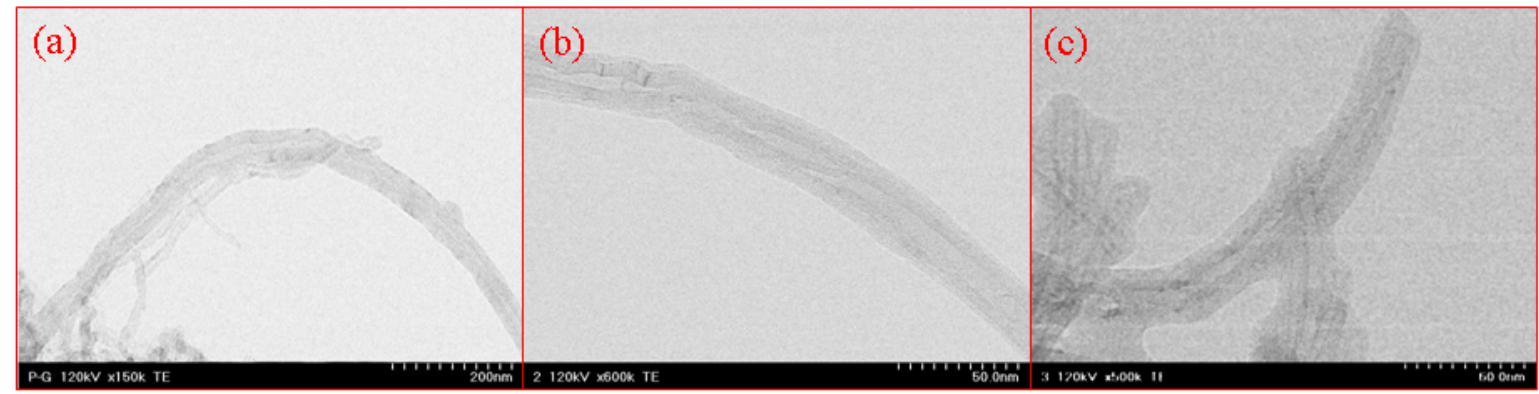

We also evaluated the morphology of MWNT ion liquid by TEM, as shown in Figure 1. The diameter of the purified MWNT was about $40 \mathrm{~nm}$, as shown in Figure 1(a); after RIGP, the diameter increased to $60 \mathrm{~nm}$ for the poly(GMA)-g-MWNT, as shown in Figure 1(b). When we introduced 1butylimidazole bromide to an epoxy group of the grafted poly(GMA), the diameter increased to $80 \mathrm{~nm}$, as shown in Figure 1(c). On the other hand, the morphology of the poly(GMA)-g-MWNT, as shown in Figure 1(b), was shown to be of tubular-type. The reason for the tubular-type morphology was considered as follows: in order to do this, we used as a vinyl monomer GMA, which is composed of hydrophilic $>\mathrm{C}=\mathrm{O}$ (carbonyl group) and $-\mathrm{C}(\mathrm{O})-\mathrm{C}$ - (epoxy group) sites and a hydrophobic vinyl group site. The vinyl group of the GMA comes to the surface of the MWNT because of a hydrophobichydrophobic interaction, while the carbonyl and epoxy group of monomer comes to the surface in an aqueous solution because of a hydrophilic-hydrophilic interaction.

Figure 2. XPS survey scan spectra of pure MWNT (a), poly(GMA)-g-MWNT (b) and MWNT ionic liquid (c).

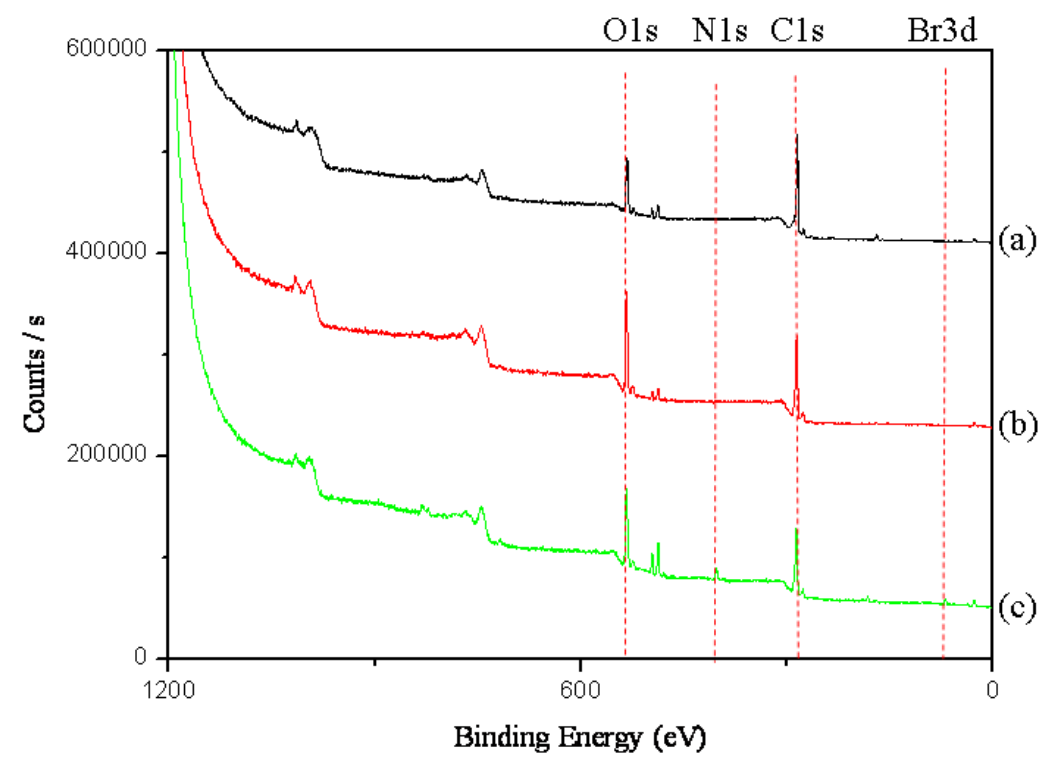


When irradiated by $\gamma$-rays, the radical polymerization of the GMA occurs on the surface of the MWNTs. Hence, we successfully obtained the tubular-type functionalized MWNT as a one-step reaction. Thus, we could easily immobilize the enzyme to the functional group of the MWNT surface by physical adsorption, and we also used the MWNT as an electron transfer material in order to increase biosensor sensitivity.

We also confirmed the successful synthesis of MWNT ion liquid via XPS spectroscopy analysis. Figure 2 shows a XPS survey scan spectra of pure MWNT (a), PGMA-g-MWNT (b) and MWNT ion liquid (c). There were significant changes after the introduction of butyl imidazolium bromide onto poly(GMA)-g-MWNT in the XPS data. The characteristic $\mathrm{Br} 3 \mathrm{~d}$ peak at $70 \mathrm{eV}$ and $\mathrm{N}$ 1s peak at $399 \mathrm{eV}$ appeared after introduction of butyl imidazolium bromide onto poly(GMA)-g-MWNT. This illustrates that the MWNT ionic liquid was successfully prepared by the RIGP method.

Figure 3. TGA curves of the purified MWNT (a), poly(GMA)-g-MWNT (b) and MWNT ionic liquid (c).

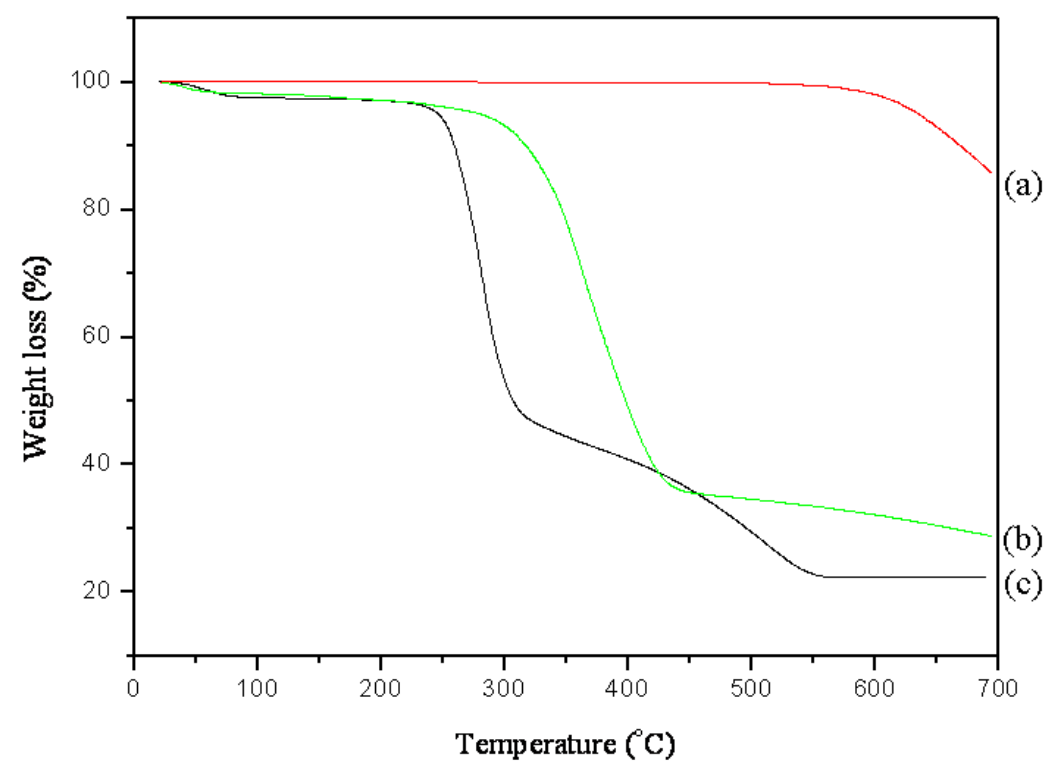

Figure 3 shows the TGA curves of the purified MWNT (a), poly(GMA)-g-MWNT and MWNT ionic liquid (c) prepared by RIGP. As shown in Figure 3, the $1^{\text {st }}$ weight loss (\%) from $50{ }^{\circ} \mathrm{C}$ to $250{ }^{\circ} \mathrm{C}$ for poly(GMA)-g-MWNT and MWNT ion liquid appeared on account of moisture because of the hydrophilic properties of the grafted poly(GMA). The $2^{\text {nd }}$ weight loss appeared in a range of $250-600{ }^{\circ} \mathrm{C}$ due to the grafted poly(GMA) weight loss. As a result, the graft yield was approximately $40 \%$ after RIGP of the GMA monomer. From these results, we confirmed the successful preparation of poly(GMA)-g-MWNT. However, $20 \%$ of MWNT remained after introduction of the imidazolium cations, as shown in Figure 3-c. As a result, it can be concluded that we were successful in the synthesis of the MWNT ionic liquid via RIGP. 


\subsection{Optimization of the Prepared MWNT Ion Liquid Electrode}

To improve the sensitivity of MWNT ion liquid toward tyrosinase, we introduced imidazolium cations to an epoxy group of poly(GMA)-g-MWNT prepared by the RIGP method. Subsequently, we fabricated a MWNT ionic liquid electrode by the hand-casting method (see Experimental section). Figure 4 reveals SEM images of the MWNT ion liquid electrode which include: ITO glass (a), ITO/chitosan (b), ITO/chitosan-tyrosinase (c), and a tyrosinase-immobilized MWNT ionic liquid electrode (d). Upon examination of the SEM images, the surface of ITO appears flat [Figure 3(a)], whereas the film of chitosan as a binder appeared as an amorphous flat form that resembles a typical polymer form, as shown in Figure 4(b). In contrast, the composite film with tyrosinase and chitosan appears as an irregular polymer form, as shown in Figure 4(c). In the MWNT ionic liquid electrode surface, the morphology of composite film changes and looks like irregular mountain patterns, as shown in Figure 4(d). As a result, we conclude that we successfully fabricated the MWNT ionic liquid electrode. The tyrosinase with plus charge strongly interacted with the anion charge of the MWNT ionic liquid. As a result, the irregular mountain morphology appeared, as shown in Figure 4(d).

Figure 4. SEM images of ITO glass (a), chitosan-ITO glass (b), tyrosinase-chitosan-ITO glass (c) and MWNT ionic liquid electrode (d).
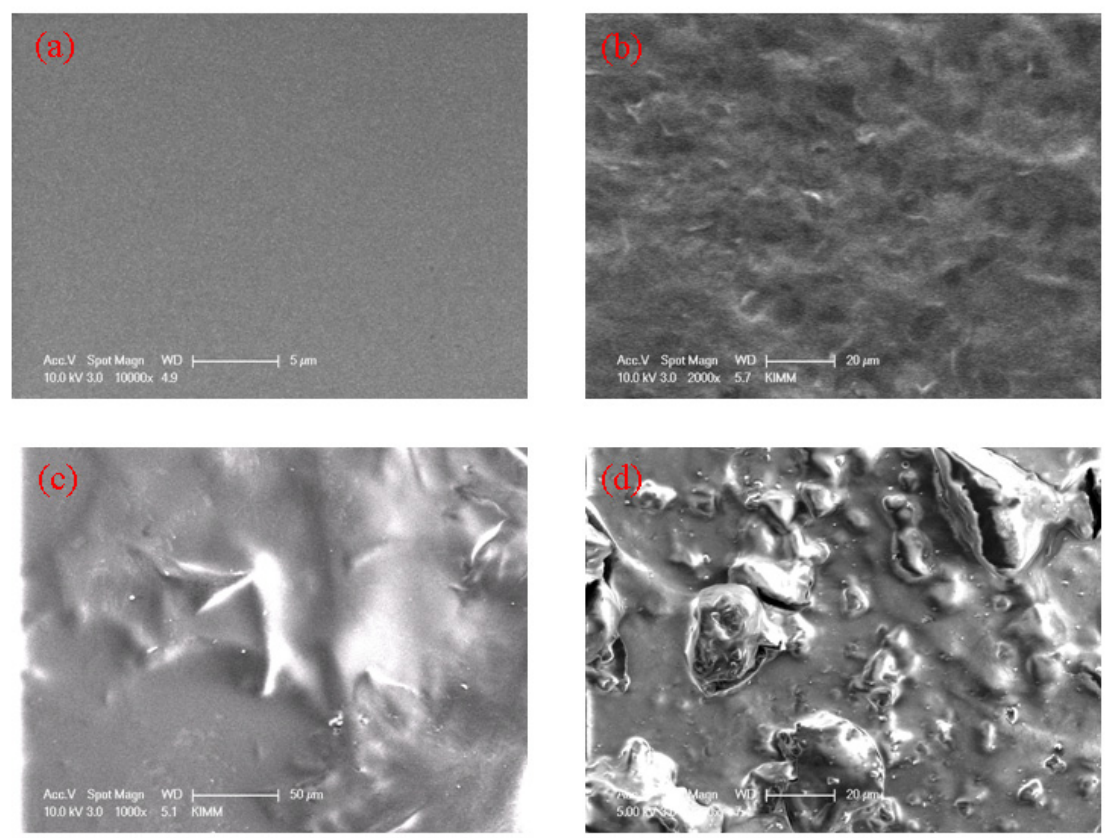

To determine the sensitivity of the MWNT ionic liquid electrode biosensor, we fabricated a tyrosinase-immobilized biosensor with or without the MWNT ionic liquid. Figure 5 shows the cyclic voltammograms of $0.5 \mathrm{mM}$ phenol in $50 \mathrm{mM}$ phosphate buffer solution using a pure MWNT electrode with tyrosinase (a), chitosan electrode with tyrosinase (b), and MWNT ion liquid electrode with tyrosinase (c). The potential is scanned between -1.0 and 0.75 versus $\mathrm{Ag} / \mathrm{AgCl}$ at a scan rate of $100 \mathrm{mVs}^{-1}$. The high oxidation peak at $-0.2 \mathrm{~V}$ and a low reduction peak at $0.2 \mathrm{~V}$ appeared on the MWNT ion liquid electrode as shown in Figure 5(c). This peak indicates the production of catechol or $o$-quinone from the enzymatic reaction in phosphate solution. There were no oxidation peaks on other 
biosensors, as shown in Figure 5(a), in spite of the high concentrations used. On the other hand, a weak oxidation peak was observed at $-0.2 \mathrm{~V}$ on the chitosan electrode with tyrosinase in Figure $5(\mathrm{~b})$. In short, tyrosinase is a copper-containing monooxygenase enzyme that catalyses the conversion of phenolic substrates to catechol and then $o$-quinones as follows:

$$
\begin{aligned}
\text { phenol }+ \text { tyrosinase }\left(\mathrm{O}_{2}\right) & \rightarrow \text { catechol } \\
\text { catecohol }+ \text { tyrosinase }\left(\mathrm{O}_{2}\right) & \rightarrow \text { o-quinone }+\mathrm{H}_{2} \mathrm{O} \\
\text { o-quinone }+\mathrm{H}^{+}+2 \mathrm{e}^{-} & \rightarrow \text { catechol }
\end{aligned}
$$

Figure 5. Cyclic voltammograms of phenol on the pure MWNT electrode with tyrosinase (a), chitosan electrode with tyrosinase (b), and MWNT ion liquid electrode with tyrosinase (c) in $2.0 \mathrm{~mL}$ phosphate buffer solution $(\mathrm{pH}=7.0)$.
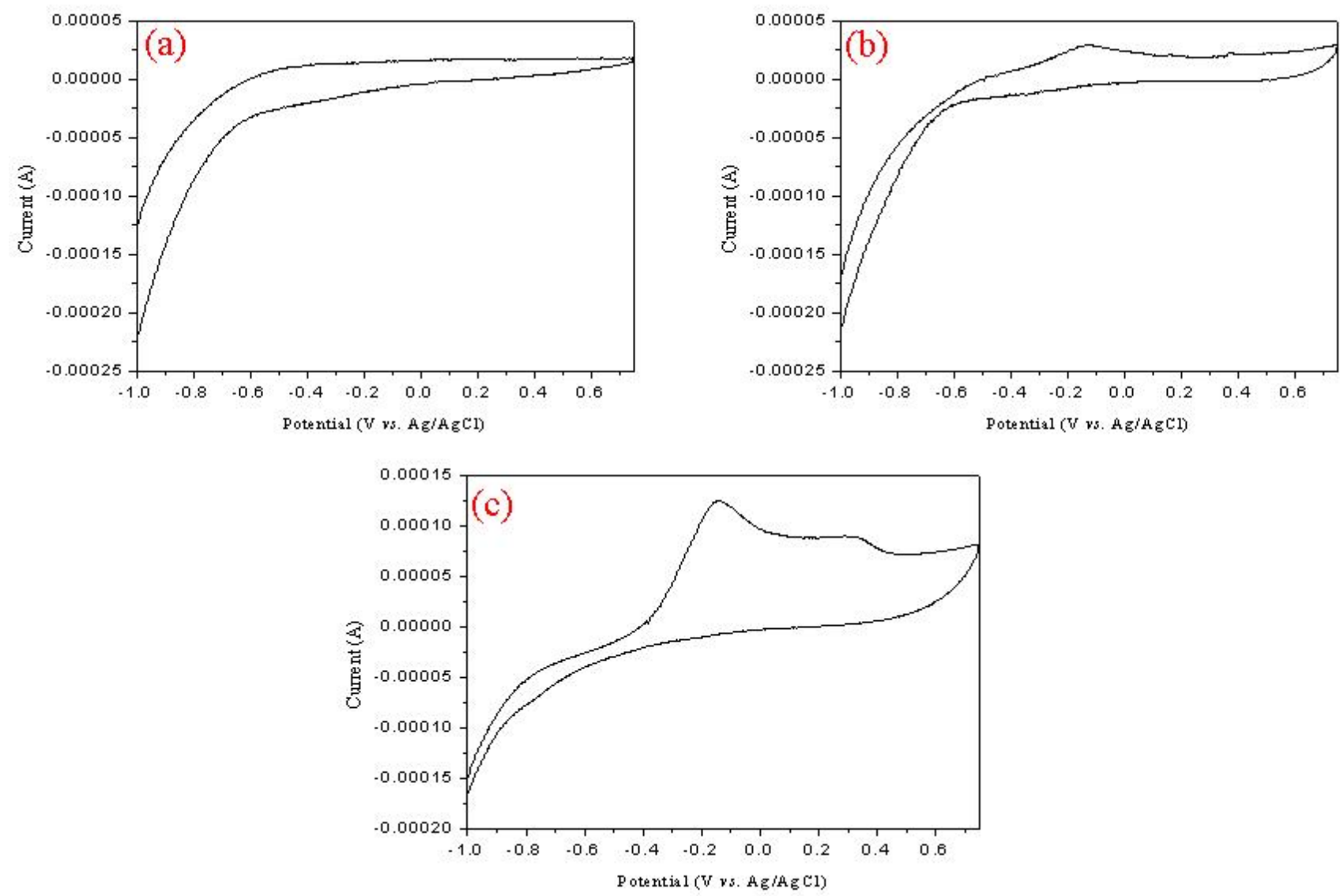

Selectivity is a very important factor in biosensors, and three compounds, phenol, $p$-cresol, and catechol, were detected by the MWNT ionic liquid electrode based on tyrosinase in a $50 \mathrm{mM}$ phosphate buffer solution ( $\mathrm{pH}=7.0$ ), as shown in Figure 6. In the $p$-cresol and catecohol solution, there was a weak redox peak for the MWNT ionic liquid electrode based on tyrosinase for this experiment. Serra et al. [21] reported that different sensitivity was observed for phenolic compounds with different substitution patterns in an enzymatic reaction. The meta-position phenol ( $m$-cresol) appeared to have a high response sensitivity as compared to ortho- and para-position phenols in an enzymatic reaction due to its high affinity for tyrosinase. As shown in Figure 6, a high response peak appeared for phenol as compared to $p$-cresol and catecohol on account of its enzymatic reaction. Tsai 
et al. [22] reported that $p$-catechol gave a high response sensitivity as compared to dopamine and ephinephrine. However, a low sensitivity response for catecohol appeared with the MWNT ionic liquid electrode based on tyrosinase.

Figure 6. Cyclic voltammograms of MWNT ionic liquid electrode based on tyrosinase in $2.0 \mathrm{~mL}$ phosphate buffer solution $(\mathrm{pH}=7.0)$ with $0.04 \mathrm{mM}$ catechol, $0.04 \mathrm{mM} p$-cresol, $0.04 \mathrm{mM}$ phenol.
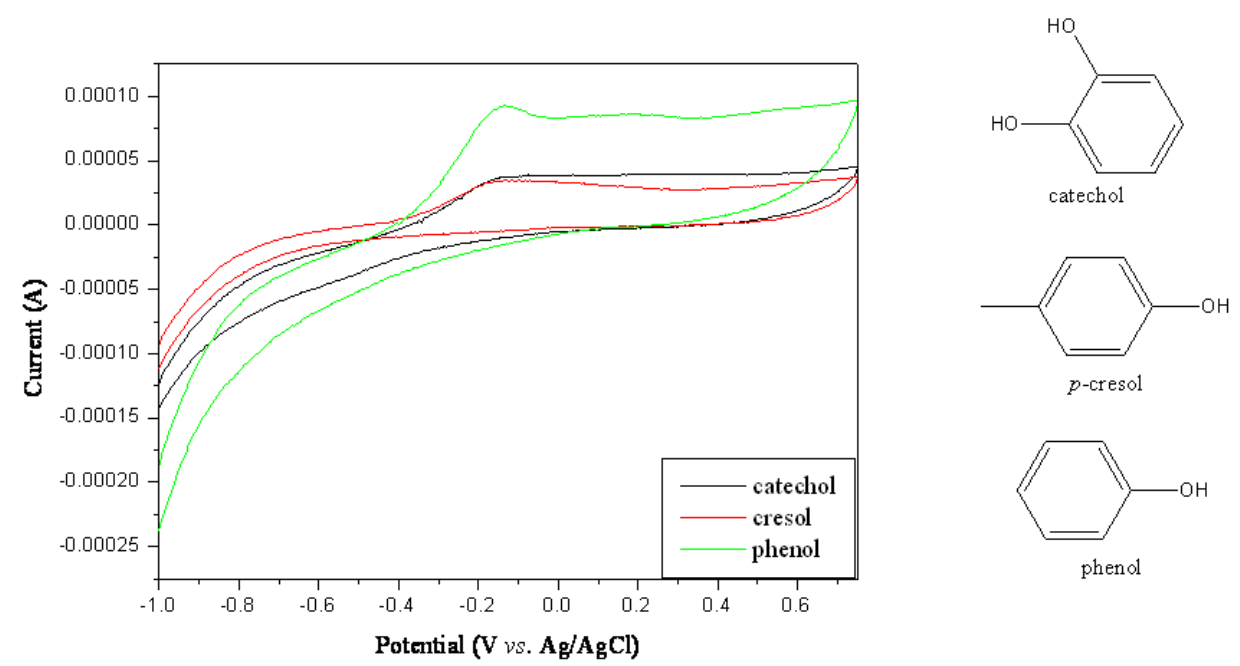

Figure 7. Cyclic voltammograms of MWNT ion liquid electrode based on tyrosinase in $2.0 \mathrm{~mL}$ phosphate buffer solution $(\mathrm{pH}=7.0)$ containing $0.01 \sim 0.08 \mathrm{mM}$ phenol (a), (b).
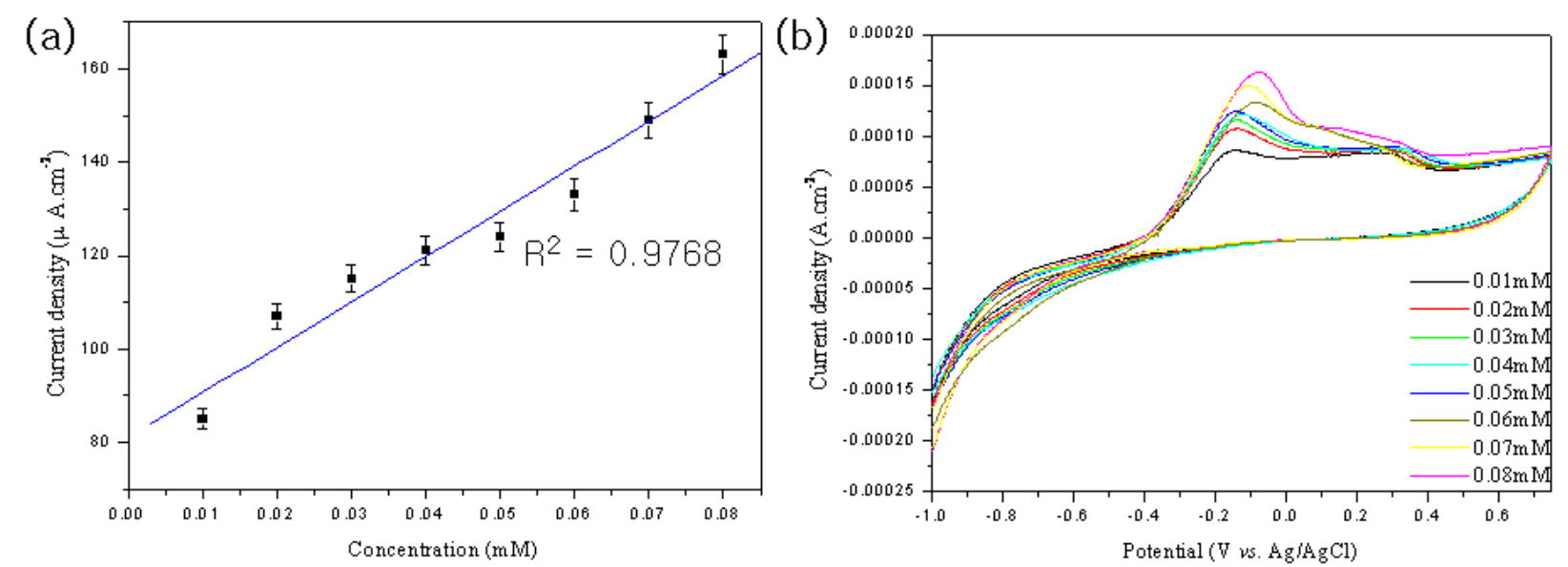

The electrochemical biosensing of phenols was performed under optimal experimental conditions. Figure 7 shows the cyclic voltammograms of phenols on the MWNT ionic liquid electrode based on tyrosinase in a $50 \mathrm{mM}$ phosphate buffer solution $(\mathrm{pH}=7.0)$ as a function of phenol concentration. The detection response range for phenol was from 0.01 to $0.08 \mathrm{mM}$ concentration, as shown in Figure 7. The sensitivity and apparent Michaelies-Menten constant $\left(\mathrm{K}_{\mathrm{M}}^{\mathrm{app}}\right)$ of the MWNT ion liquid based on tyrosinase were $2.925 \mathrm{~A}\left(\mathrm{~mol} \cdot \mathrm{L}^{-1}\right)^{-1}$ and $(0.041) \mathrm{mmol} \cdot \mathrm{L}^{-1}$, respectively. The $\mathrm{K}_{\mathrm{M}}{ }^{\text {app }}$ values were lower than those reported for the free enzymes in solution, which was estimated to be 700 $\mu \mathrm{mol} \cdot \mathrm{L}^{-1}$ as a substrate [23]. Such low $\mathrm{K}_{\mathrm{M}}{ }^{\text {app }}$ values may be attributed to the high concentration of 
tyrosinase on the biosensor. In addition, the embedded functionalized MWNT in a composite film may have increased the access of substrate molecules to enzyme catalytic sites.

Another parameter affecting the sensing efficiency of the MWNT ionic liquid electrode based on tyrosinase is the $\mathrm{pH}$ of the supporting electrolyte. Figure 8 presents cyclic voltammograms of $0.04 \mathrm{mM}$ phenol on the MWNT ionic liquid electrode based on tyrosinase in $50 \mathrm{mM}$ phosphate buffer solution as a function of $\mathrm{pH}$. The sensing efficiency increased with increasing $\mathrm{pH}$ levels from 4.0 to 7.0 , and then rapidly decreased with increasing $\mathrm{pH}$ levels, as shown in Figure 8. This means that at low $\mathrm{pH}$ levels, the sensing efficiency of biosensors is due to enzyme activity.

Figure 8. Response sensitivity of the MWNT ion liquid electrode based on tyrosinase as function of $\mathrm{pH}$ value for $0.04 \mathrm{mM}$ phenol in $2 \mathrm{~mL}$ phosphate buffer (a), (b).
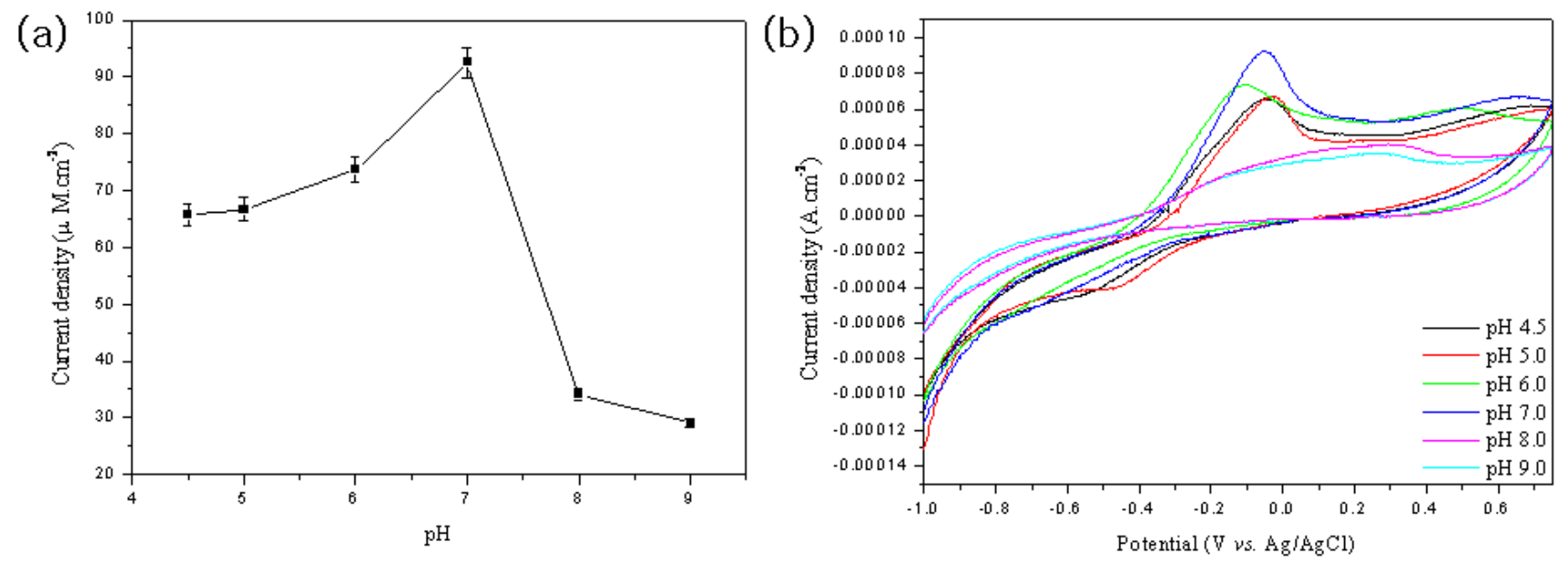

Figure 9. Response sensitivity of the MWNT ion liquid electrode based on tyrosinase as a function of temperature for $0.04 \mathrm{mM}$ phenol in $2.0 \mathrm{~mL}$ phosphate buffer (a), (b).

(a)

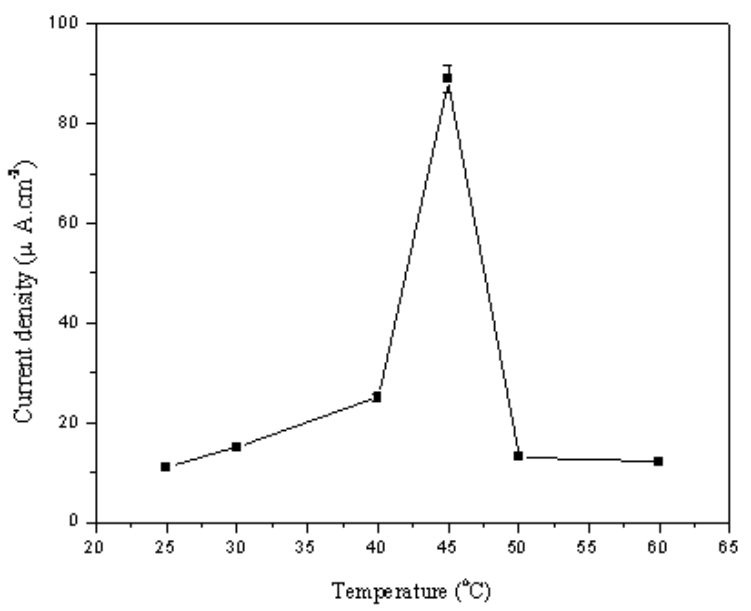

(b)

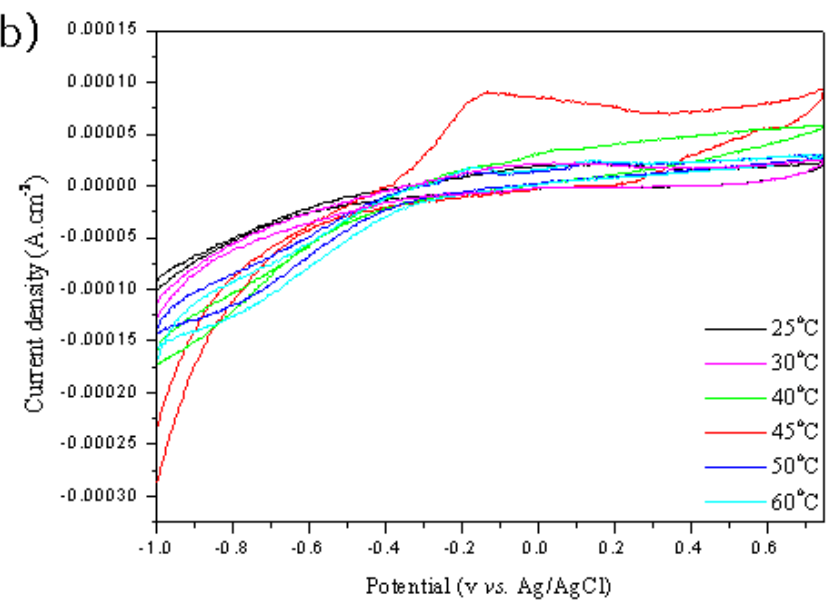

The effect of temperature on the amperometric response of phenol was also studied and is shown in Figure 9. Prior to cyclic voltammetry detection, the MWNT ionic liquid electrode based on tyrosinase was immersed into a buffer solution at a given temperature for 10 minutes, and then the CV was recorded. As shown in Figure 9, the response sensitivity increased with increasing temperatures 
from 20 to $45^{\circ} \mathrm{C}$, and then rapidly decreased when temperatures were increased. Maximum sensitivity was attained at $45{ }^{\circ} \mathrm{C}$. At high temperatures, the response sensitivity of the MWNT ionic liquid electrode based on tyrosinase for phenol rapidly decreased due to partial breakdown of the immobilized tyrosinase. Although the response sensitivity of the biosensor was greatest at $45{ }^{\circ} \mathrm{C}$, considering that most enzymes can be easily broken down at high temperatures and the practical application that requires a simple experimental procedure and the long lifetime of the biosensor, room temperature was used consistently throughout the study.

\subsection{Total Amounts of Phenolics in Commercial Red Wines}

Three red wines: Brand Chateau Mani-dry, Chateau Mani-sweet, Chateau Mani-nouveau, made in Korea, were used in the analysis of phenolic compound amounts in wines. Figure 10 shows cyclic voltammograms of the MWNT ionic liquid electrode based on tyrosinase in a $2.0 \mathrm{~mL}$ phosphate buffer solution ( $\mathrm{pH}=7.0$ ) containing $8.0 \mu \mathrm{L}$ Chateau Mani-dry (a), 8.0 $\mu \mathrm{L}$ Chateau Mani-sweet (b), $8.0 \mu \mathrm{L}$ Chateau Mani-nouveau (c), respectively, and in $2.0 \mathrm{~mL}$ Chateau Mani-dry (d), 2.0 mL Chateau Manisweet (e), $2.0 \mathrm{~mL}$ Chateau Mani-nouveau (f) plain red wine. As shown in Figure 10(a-c), the oxidation peak of red wine in a phosphate buffer using the MWNT ionic liquid electrode appeared at $-0.2 \mathrm{~V}$, while the phenol peak in plain red wine without a phosphate buffer shifted to a plus potential in Figure 10(d-f).

Figure 10. Cyclic voltammograms of MWNT ion liquid electrode based on tyrosinase in $2.0 \mathrm{~mL}$ phosphate buffer solution ( $\mathrm{pH}=7.0$ ) containing $8 \mathrm{~mL}$ Chateau Mani-dry (a), Chateau Manisweet (b), Chateau Mani-nouveau (c) and in $2.0 \mathrm{~mL}$ Chateau Mani-dry (d), Chateau Mani-sweet (e), Chateau Mani-nouveau (f) as electrolyte.
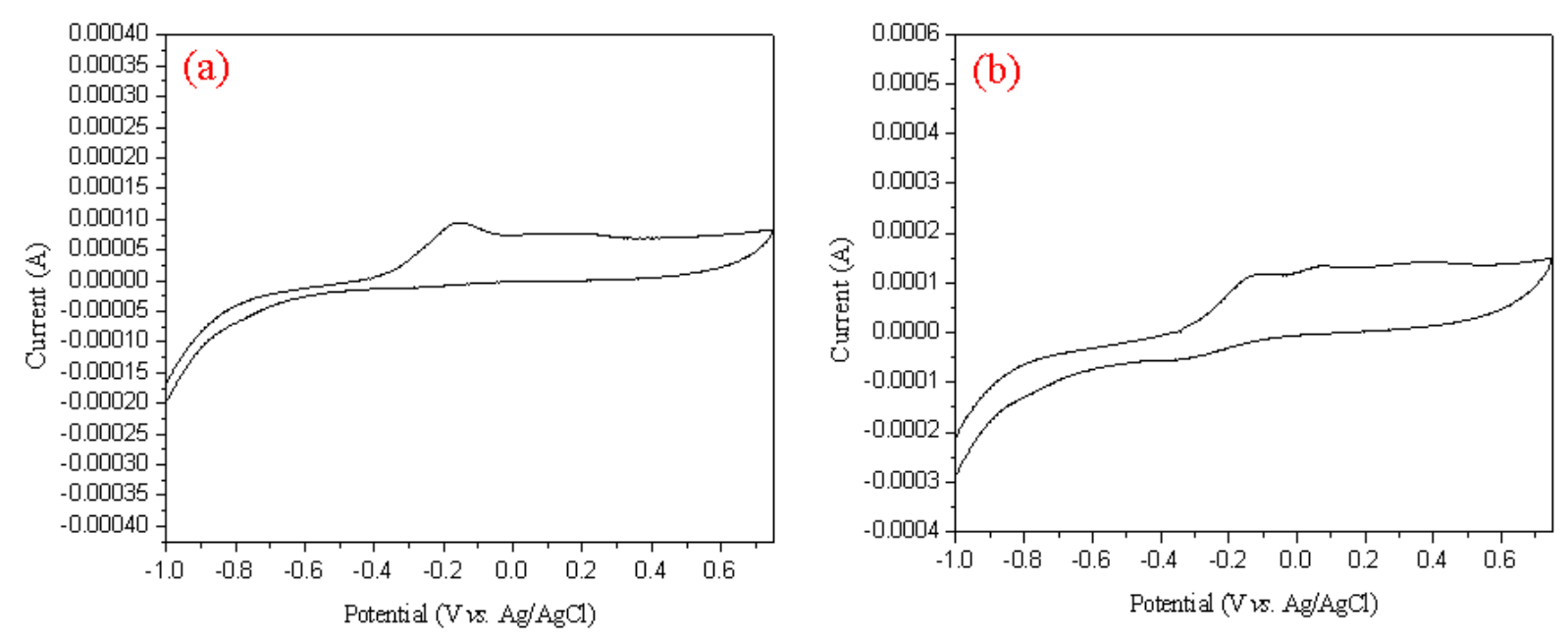
Figure 10. Cont.
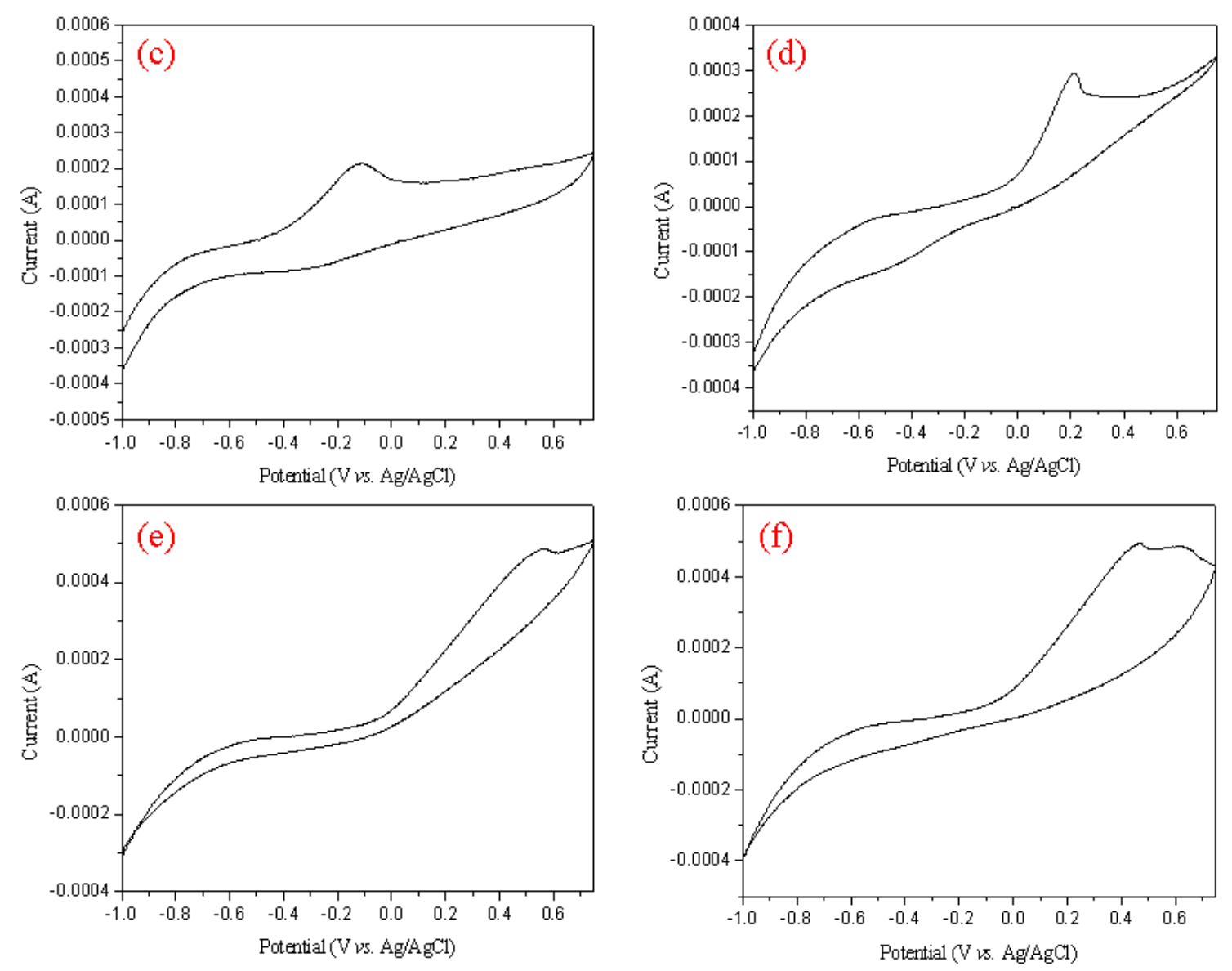

Table 2. Total phenolic concentration determined by the MWNT ionic liquid electrode based on tyrosinase for commercial red wines.

\begin{tabular}{lcll}
\hline Commercial red wine (Korea) & Current density & Phenolics $^{\text {a) }}$ & Phenolics $^{\text {b) }}$ \\
\hline Chateau Mani-dry (Korea) & $9.7 \times 10^{-5} \mathrm{~A}$ & $383.5 \mathrm{mg} / \mathrm{L}$ & $20.33 \mathrm{mg} / \mathrm{L}$ \\
Chateau Mani-sweet (Korea) & $1.2 \times 10^{-4} \mathrm{~A}$ & $872.9 \mathrm{mg} / \mathrm{L}$ & $38.02 \mathrm{mg} / \mathrm{L}$ \\
Chateau Mani-nouveau (Korea) & $2.1 \times 10^{-4} \mathrm{~A}$ & $3087 \mathrm{mg} / \mathrm{L}$ & $39.01 \mathrm{mg} / \mathrm{L}$ \\
\hline
\end{tabular}

a) in phosphate buffer containing $8 \mathrm{~mL}$ commercial red wine. b) in crude red wine of $2.0 \mathrm{~mL}$.

Table 2 summarizes the total phenolics in a phosphate buffer and plain red wine on the MWNT ionic liquid electrode based on tyrosinase at room temperature. Table 2 presents the amount of phenolics in commercial red wines in the range of $383.5-3,087 \mathrm{mg} / \mathrm{L}$ in a phosphate buffer solution.

\section{Conclusions}

In this study, we fabricated an immobilized tyrosinase biosensor based on a MWNT ionic liquid prepared by radiation-induced graft polymerization. The sensing range of the tyrosinase-immobilized biosensor based on MWNT ionic liquid for phenol was from 0.01 to $0.08 \mathrm{mM}$. The tyrosinaseimmobilized biosensor based in the MWNT ionic liquid was optimimized for $\mathrm{pH}$, temperature, and other phenolic compounds. The prepared biosensor was used to determine phenolics in commercial red 
wines. As a result, the amount of phenolics in commercial red wines have been determined to be in the range of $383.5^{-3,087} \mathrm{mg} / \mathrm{L}$ in a phosphate buffer solution. This range was calculated from a calibration curve of phenols on a tyrosinase-immobilized biosensor based on a MWNT ionic liquid electrode. The high amounts of phenolic compounds in Brand Chateau Mani-nouveau appear to be responsible for the bitter taste of this red wine.

\section{Acknowledgements}

This research was supported by Nano R\&D program and The National Nuclear Technology program through the Korea Science and Engineering Foundation funded by the Ministry of Science \& Technology. This research was partly supported by the Hannam University Research Fund (2009). The authors would like to thank the Korea Basic Science Institute (KBSI) for FETEM measurements.

\section{References and Notes}

1. Wang, J.; Musameh, M. Carbon Nanotube/Teflon Composite Electrochemical Sensors and Biosensors. Anal. Chem. 2003, 75, 2075-2079.

2. Wang, J.; Musameh, M.; Lin, Y.H. Solubilization of Carbon Nanotubes by Nafion toward the Preparation of Amperometric Biosensors. J. Am. Chem. Soc. 2003, 125, 2408-2409.

3. Saito, Y.; Yoshihawa, T. Carbon Nanocapsules Encaging Metals and Carbides. J. Phys. Chem. Solids. 1993, 54, 1849-1860.

4. Compton, D.L.; Laszlo, J.A. Direct Electrochemical Reduction of Hemin in Imidazoliumbasedionic Liquids. J. Electroanal. Chem. 2002, 520, 71-78.

5. Sweeny, B.K.; Peters, D.G. Cyclic Voltammetric Study of the Catalytic Behavior of Nickel(I) Salen Electrogenerated at a Glassy Carbon Electrode in an Ionic Liquid (1-butyl-3methylimidazolium tetrafluoroborate, $\mathrm{BMIM}^{+} \mathrm{BF}_{4}{ }^{-}$). Electrochem. Commun. 2001, 3, 712-715.

6. Zhao, F.; Wu, X.; Wang, M.; Lin, Y.; Gao, L.; Dong, S. Electrochemical and Bioelectrochemistry Properties of Room-Temperature Ionic Liquids and Carbon Composite Materials. Anal. Chem. 2004, 76, 4960-4967.

7. Li, J.; Yu, J.; Zhao, F.; Zeng, B. Direct Electrochemistry of Glucose Oxidase Entrapped in Nano Gold Particles-ionic Liquid- $N, N$-dimethylformamide Composite Film on Glassy Carbon Electrode and Glucose Sensing. Anal. Chem. Acta 2007, 587, 33-40.

8. Sun, W.; Wang, D.; Gao, R.; Jiao, K. Direct Electrochemistry and Electrocatalysis of Hemoglobin in Sodium Alginate Film on a BMIMPF 6 Modified Carbon Paste Electrode. Electrochem. Commun. 2007, 9, 1159-1164.

9. Safavi, A.; Maleki, N.; Moradlou, O.; Sorouri, M. Direct Electrochemistry of Hemoglobin and Its Electrocatalytic Effect based on Its Direct Immobilization on Carbon Ionic Liquid Electrode. Electrochem. Commun. 2008, 10, 420-423.

10. Choi, S.-H.; Lee, K.P.; Lee, J.G. Adsorption Behavior of Urokinase by Polypropylene Film Modified with Amino Acid as Affinity Group. Microchem. J. 2001, 68, 205-213. 
11. Choi, S.-H.; Jeong, Y.H.; Ryoo, J.J.; Lee, K.P. Desalination by Electrodialysis with Ion-Exchange Membrane Prepared by Radiation-induced Graft Polymerization. Radiati. Phys. Chem. 2001, 60, 503-511.

12. Choi, S.-H.; Kang, H.J.; Ryu, E.N.; Lee, K.P. Electrochemical Properties of Polyolefin Nonwoven Fabric Modified with Carboxylic Acid Group for Battery Separator. Radiati. Phys. Chem. 2001, 60, 495-502.

13. Yang, D.-S.; Jung, D.-J.; Choi, S.-H. One-step Functionalization of Multi-walled Carbon Nanotube by Radiation-induced Graft Polymerization and Their Application of Enzyme-free Biosensors. Reac. Func. Polym. (accepted).

14. Saito, K.; Kaga, T.; Yamagishi, H.; Furusaki, S. Phosphorylated Hollow Fibers Synthesized by Radiation Grafting and Cross-linking. J. Membr. Sci. 1989, 43, 131-141.

15. Choi, S.H.; Nho, Y.C.; Kim, G.T. Adsorption of $\mathrm{Pb}^{2+}$ and $\mathrm{Pd}^{2+}$ on Polyethylene Membrane with Amino Group Modified by Radiation-Induced Graft Copolymerization. J. Appl. Polym. Sci. 1999, 71, 643-650.

16. Choi, S.H.; Nho, Y.C. Adsorption of $\mathrm{Pb}^{2+}, \mathrm{Cu}^{2+}$, and $\mathrm{Co}^{2+}$ by Polypropylene Fabric and Polyethylene Hollow Fiber Modified by Radiation-Induced Graft Copolymerization. J. Chem. Eng. 1999, 16, 241-247.

17. Choi, S.H.; Nho, Y.C. Adsorption of $\mathrm{Co}^{2+}$ by Styrene-g-polyethylene Membrane Bearing Sulfonic Acid Group Modified by Radiation-Induced Graft Copolymerization. Kor. J. Appl. Polym. Sci. 1999, 71, 2227-2235.

18. Choi, S.H.; Nho, Y.C. Modification of Hollow Fiber Membrane with Amidoxime, Iminodiacetic Acid and Diethylene triamine by Radiation-Induced Graft Copolymerization. Kor. Polym. J. 1999, 7, 38-45.

19. Campanella, L.; Bonanni, A.; Finotti, E.; Tomasetti, M. Biosensors for Determination of Total and Natural Antioxidant Capacity of Red and White Wines: Comparison with other Spectrophotometric and Fluorimetric Methods. Biosens. Bioelectron. 2004, 19, 641-651.

20. Luximon-Ramma, A.; Bahorun, T.; Crozier, A.; Zbarsky, V.; Datla, K.P.; Dexter, D.T. Characterization of the Antioxidant Functions of Flavonoids and Proanthocyanidins in Mauritian Black Teas. Food Res. Int. 2005, 38, 357-367.

21. Serra, B.; Jimenez, S.; Mena, M.L.; Reviejo, A.J.; Pingarron, J.M. Composite Electrochemical Biosensors: A Comparison of Three Different Electrode Matrices for the Construction of Amperometric Tyrosinase Biosensors. Biosens. Bioelectron. 2002, 17, 217.

22. Tsai, Y.-C.; Chiu, C.-C. Amperometric Biosensors based on Multiwalled Carbon NanotubeNafion-tyrosinase Nanobiocomposites for the Determination of Phenolic Compounds. Sens. Actuats. B: Chem. 2007, 125, 10-16.

23. Espin, J.C.; Varon, R.; Fenoll, L.G.; Gilabert, M.A.; Garcia-Ruiz, P.A.; Tudele, J.; Canovas, F. Kinetic Characterization of the Substrate Specificity and Mechanism of Mushroom Tyrosinase. Eur. J. Biochem. 2000, 22, 1270-1279.

(C) 2009 by the authors; licensee Molecular Diversity Preservation International, Basel, Switzerland. This article is an open-access article distributed under the terms and conditions of the Creative Commons Attribution license (http://creativecommons.org/licenses/by/3.0/). 